HUPD-1212

\title{
Regularization parameter independent analysis in Nambu-Jona-Lasinio model
}

\author{
T. Inagaki \\ Information Media Center, Hiroshima University, Higashi-Hiroshima, Hiroshima 739-8521, Japan \\ D. Kimura \\ Faculty of Engineering, Kinki University, Higashi-Hiroshima, Hiroshima, 739-2116, Japan \\ H. Kohyama \\ Department of Physics, Kyungpook National University, Daegu 702-701, Korea
}

A. Kvinikhidze

A. Razmadze Mathematical Institute of Georgian Academy of Sciences, M. Alexidze Str. 1, 380093 Tbilisi, Georgia

(Dated: September 25, 2018)

\begin{abstract}
Nambu-Jona-Lasinio model used to investigate low energy phenomena is nonrenormalizable, therefore the results depend on the regularization parameter in general. A possibility of the finite in four-dimensional limit and even the regularization parameter (this is dimension in the dimensional regularization scheme) independent analysis is shown in the leading order of the $1 / N_{c}$ expansion.
\end{abstract}

PACS numbers: 11.30.Qc, 12.39.-x

\section{INTRODUCTION}

Nambu-Jona-Lasinio (NJL) model [1] is one of the most popular QCD motivated effective theories used to understand non-perturbative low energy phenomena of strong interactions [2 4].

Its Lagrangian contains a four-fermion interaction, an operator whose dimension exceeds the space-time dimension, $D$, for $D>2$. It is known that the four-fermion interaction model is renormalizable in the $1 / N_{c}$ expansion scheme for $2<D<4$ and the model possesses an ultraviolet-stable point [6-8]. It is nonrenormalizable in four space-time dimensions, however, the ultraviolet divergences remaining in the renormalized Green's function are logarithmic in the leading order of the $1 / N_{c}$ expansion [7].

In order to construct an effective theory out of infinite number of operators involved in the NJL model one usually picks up operators which make a major contribution to targeted phenomena. For example, scalar type four-fermion operators are considered.

As the NJL model is not renormalizable in four spacetime dimensions some regularization methods are used to avoid divergences of loop integrals thereby to obtain finite values of predicted physical quantities.

Three-momentum sharp cutoff regularization is widely used. Other regularization procedures are also studied in NJL type models, e.g., the smooth cutoff [9, 10], the dimensional regularization (DR) [11 18], the PauliVillars [19] and the Fock-Schwinger proper-time regularization [20, 21]. The regularization parameter dependence is discussed in the three-momentum sharp cutoff and in the DR schemes in [15 18].

In this paper we show that for some quantities the NJL model behaves well in the ultraviolet limit. Moreover, the predicted quantities have reasonable values in the $4 \mathrm{D}$ limit; they are close to those obtained in the DR scheme where the dimension $D<4$ is a regularisation parameter.

The paper is organized as follows. In Sec. II. the threeflavor NJL model introduced. Then we briefly review the dimensional regularization. In Sec. III, we calculate meson masses, their decay constants, etc., in the leading order of the $1 / N_{c}$ expansion using DR. In Sec. IV, the regularization parameter dependence is discussed. In Sec. V. the four dimensional limit is considered. The order of divergences is evaluated for $n$ point Green functions in the leading order of the $1 / N_{c}$ expansion. Finite values are obtained for physical observables. We also derive analytic relationships between observables. In Sec. VI, we phenomenologically fix the model parameters and numerically evaluate the physical quantities. Some concluding remarks are given in Sec. VII.

\section{NJL MODEL}

\section{A. NJL model}

The three-flavor NJL model including KobayashiMaskawa-'t Hooft term [22, 23] is given as,

$$
\mathcal{L}_{\mathrm{NJL}}=\sum_{i, j} \bar{q}_{i}(i \not \partial-\hat{m})_{i j} q_{j}+\mathcal{L}_{4}+\mathcal{L}_{6},
$$

where

$$
\begin{aligned}
& \mathcal{L}_{4}=G \sum_{a=0}^{8}\left[\left(\sum_{i, j} \bar{q}_{i} \lambda_{a} q_{j}\right)^{2}+\left(\sum_{i, j} \bar{q}_{i} i \gamma_{5} \lambda_{a} q_{j}\right)^{2}\right], \\
& \mathcal{L}_{6}=-K\left[\operatorname{det} \bar{q}_{i}\left(1-\gamma_{5}\right) q_{j}+\text { H.c. }\right]
\end{aligned}
$$


the subscripts $i, j$ are the flavor indices, $i, j=u, d, s$, and $\hat{m}$ denotes the current quark mass matrix, $\hat{m}=$ $\operatorname{diag}\left(m_{u}, m_{d}, m_{s}\right)$. Below we consider the $\mathrm{SU}(2)$ isospin symmetric case, $m_{u}=m_{d}$, for simplicity. $\lambda_{a}$ are the Gell-Mann matrices in the flavor space, $G$ and $K$ represent the effective coupling constants for four- and sixfermion interaction, respectively. $G$ and $K$ have negative mass dimensions, -2 and -5 respectively, so the model is nonrenormalizable in four space-time dimensions. The determinant in $\mathcal{L}_{6}$ concerns the matrix elements labeled by the flavor indices. We suppose the order of the coupling constants to be $G N_{c} \simeq O(1)$ and $K N_{c}^{2} \simeq O(1)$, where $N_{c}$ is the number of colors.

The chiral condensates $\langle\bar{i} i\rangle$ generate the constituent quark masses, $m_{i}^{*}$, inside mesons. One solves the gap equations to evaluate the constituent quark masses. In the leading order of $1 / N_{c}$ expansion, the gap equations are obtained as follows [2 4],

$$
m_{i}^{*}=m_{i}+4 G\left(i \operatorname{tr} S^{i}\right)+2 K\left(i \operatorname{tr} S^{j}\right)\left(i \operatorname{tr} S^{k}\right),
$$

with $i \neq j \neq k \neq i . \operatorname{tr} S^{i}$ represent the chiral condensates, $i \operatorname{tr} S^{i}=-\langle\bar{i} i\rangle$ which are given by the trace of the quark propagator,

$$
i \operatorname{tr} S^{i}=-\int \frac{d^{D} p}{i(2 \pi)^{D}} \operatorname{tr} S^{i}(p)
$$

where

$$
S^{i}(p) \equiv \frac{1}{\not p-m_{i}^{*}+i \varepsilon}
$$

and $D(\equiv 4-2 \epsilon)$ is the space-time dimensions for internal quark fields. "tr" in the integral denotes the trace with respect to the spinor and color indices.

\section{B. Dimensional regularization}

The quark loop integral in Eq. (5) is divergent in four space-time dimensions. One has to regularize it to obtain a finite result. The regularization dependence for the physical quantities are induced by this procedure. Using the DR we are going to take the four space-time dimensional limit.

In the DR we have

$$
i \operatorname{tr} S^{i}=\frac{N_{c}}{(2 \pi)^{D / 2}} \Gamma\left(1-\frac{D}{2}\right) m_{i}^{*}\left(m_{i}^{* 2}\right)^{D / 2-1} .
$$

where the mass dimension is a function of $D$. The integral like Eq. (7), needs to be multiplied by the mass scale parameter $M_{0}^{4-D}$ in order to correct the mass dimension. Note that in the previous studies [16 18], this parameter is called "the renormalization scale". However, in this paper, we name it "mass scale parameter" to clearly distinguish our treatment from the renormalization. Then, the "rescaled" chiral condensates are given by

$$
\langle\bar{i} i\rangle_{\mathrm{rs}}=M_{0}^{4-D}\langle\bar{i} i\rangle .
$$

It is also important to discuss the mass dimension of the coupling constants $G$ and $K$. Substituting Eq. (7) into the gap equations (4) tells us that the mass dimensions of $G$ and $K$ are $2-D$ and $3-2 D$, respectively. Then the rescaled couplings are evaluated as

$$
G_{\mathrm{rs}}=M_{0}^{D-4} G, \quad K_{\mathrm{rs}}=M_{0}^{2(D-4)} K .
$$

It is worth mentioning that $G \operatorname{tr} S$ and $K(\operatorname{tr} S)^{2}$ do not depend on $M_{0}$, so the constituent quark masses $m_{i}^{*}$ are independent of $M_{0}$.

This mass-rescaling parameter plays a key role to systematically control the divergences of the loop integrals. We will show how the model can produce physical quantities in the ultraviolet limit.

\section{PHYSICAL QUANTITIES}

We present the prescription to calculate meson masses, meson decay constants and topological susceptibility in the leading order of the $1 / N_{c}$ expansion. These quantities are derived from four-, two-point functions and bubble diagrams, respectively.

\section{A. Pion and Kaon masses}

The masses of pion and kaon are obtained by evaluating the poles of their propagators

$$
\Delta_{\mathrm{P}}\left(k^{2}\right)=\frac{2 K_{\alpha}}{1-2 K_{\alpha} \Pi_{\mathrm{P}}\left(k^{2}\right)},
$$

using the random-phase approximation and the $1 / N_{c}$ expansion, where $\alpha$ labels the channel isospin and $\mathrm{P}$ denotes the meson species. The explicit form of the flavordependent effective couplings $K_{\alpha}$ is given by

$$
\begin{aligned}
& K_{3} \equiv G+\frac{1}{2} K\left(i \operatorname{tr} S^{s}\right), \quad \text { for } \quad \pi^{0}, \\
& K_{6} \equiv G+\frac{1}{2} K\left(i \operatorname{tr} S^{u}\right), \quad \text { for } \quad K^{0}, \overline{\mathrm{K}}^{0} .
\end{aligned}
$$

$\Pi_{\mathrm{P}}$ is the meson self-energy,

$$
\begin{aligned}
& \Pi_{\pi}\left(k^{2}\right)=2 \Pi_{5}^{u u}\left(k^{2}\right), \\
& \Pi_{\mathrm{K}}\left(k^{2}\right)=2 \Pi_{5}^{s u}\left(k^{2}\right),
\end{aligned}
$$

where $\Pi_{5}^{i j}\left(k^{2}\right)$ is the following loop integral:

$$
\begin{aligned}
\Pi_{5}^{i j}\left(k^{2}\right) & =\int \frac{d^{D} p}{i(2 \pi)^{D}} \operatorname{tr}\left[\gamma_{5} S^{i}(p+k / 2) \gamma_{5} S^{j}(p-k / 2)\right] \\
= & \frac{1}{2}\left(\frac{i \operatorname{tr} S^{i}}{m_{i}^{*}}+\frac{i \operatorname{tr} S^{j}}{m_{j}^{*}}\right)+\frac{1}{2}\left[k^{2}-\left(m_{i}^{*}-m_{j}^{*}\right)^{2}\right] I_{i j}\left(k^{2}\right),
\end{aligned}
$$




$$
I_{i j}\left(k^{2}\right)=\int \frac{d^{D} p}{i(2 \pi)^{D}} \frac{\operatorname{tr} 1}{\left(p^{2}-m_{i}^{* 2}\right)\left[(p-k)^{2}-m_{j}^{* 2}\right]} .
$$

The trace runs over color and spinor indices, then $\operatorname{tr} 1=$ $2^{D / 2} N_{c}$.

The conditions which determine the pion and kaon masses are

$$
\begin{aligned}
& 1-2 K_{3} \Pi_{\pi}\left(m_{\pi}^{2}\right)=0, \\
& 1-2 K_{6} \Pi_{\mathrm{K}}\left(m_{\mathrm{K}}^{2}\right)=0 .
\end{aligned}
$$

These equations are the relations between model parameters and the input physical quantities $m_{\pi}$ and $m_{\mathrm{K}}$.

\section{B. $\quad \eta$ and $\eta^{\prime}$ masses}

$\eta$ and $\eta^{\prime}$ mesons are the mass eigenstates for $\eta_{8}$ and $\eta_{0}$ mixing. In the random-phase approximation and the $1 / N_{c}$ expansion, the propagator of the $\eta-\eta^{\prime}$ system is given by [3, 4]

$$
\boldsymbol{\Delta}^{+}\left(k^{2}\right)=\frac{2 \boldsymbol{K}^{+}}{1-2 \boldsymbol{K}^{+} \boldsymbol{\Pi}\left(k^{2}\right)},
$$

where the effective coupling $\boldsymbol{K}^{+}$and the self-energy $\boldsymbol{\Pi}$ are the $2 \times 2$ matrices

$$
\begin{aligned}
\boldsymbol{K}^{+} & =\left(\begin{array}{ll}
K_{00} & K_{08} \\
K_{80} & K_{88}
\end{array}\right), \\
\boldsymbol{\Pi} & =\left(\begin{array}{ll}
\Pi_{00} & \Pi_{08} \\
\Pi_{80} & \Pi_{88}
\end{array}\right),
\end{aligned}
$$

with

$$
\begin{aligned}
& K_{00}=G-\frac{1}{3} K\left(i \operatorname{tr} S^{s}+2 i \operatorname{tr} S^{u}\right), \\
& K_{88}=G-\frac{1}{6} K\left(i \operatorname{tr} S^{s}-4 i \operatorname{tr} S^{u}\right), \\
& K_{08}=K_{80}=-\frac{\sqrt{2}}{6} K\left(i \operatorname{tr} S^{s}-i \operatorname{tr} S^{u}\right),
\end{aligned}
$$

and

$$
\begin{aligned}
& \Pi_{00}\left(k^{2}\right)=\frac{2}{3}\left[2 \Pi_{5}^{u u}\left(k^{2}\right)+\Pi_{5}^{s s}\left(k^{2}\right)\right], \\
& \Pi_{88}\left(k^{2}\right)=\frac{2}{3}\left[\Pi_{5}^{u u}\left(k^{2}\right)+2 \Pi_{5}^{s s}\left(k^{2}\right)\right], \\
& \Pi_{08}\left(k^{2}\right)=\Pi_{80}\left(k^{2}\right)=\frac{2 \sqrt{2}}{3}\left[\Pi_{5}^{u u}\left(k^{2}\right)-\Pi_{5}^{s s}\left(k^{2}\right)\right] .
\end{aligned}
$$

The masses of $\eta$ and $\eta^{\prime}$ are obtained by solving

$$
\operatorname{det}\left[1-2 \boldsymbol{K}^{+} \boldsymbol{\Pi}\left(m_{\mathrm{P}}^{2}\right)\right]=0,
$$

where $m_{\mathrm{P}}$ denotes the mass of $\eta$ or $\eta^{\prime}$. They are also obtained via diagonalization of Eq. (19) [4, 25].

\section{Pion and Kaon decay constants}

The decay constants of pion and kaon, $f_{\mathrm{P}}$ are defined by the matrix element of axial current between the meson and vacuum states,

$$
\begin{aligned}
& i k_{\mu} f_{\mathrm{P}} \delta_{\alpha \beta} \\
& =-M_{0}^{4-D} \int \frac{d^{D} p}{(2 \pi)^{D}} \operatorname{tr}\left[\gamma_{\mu} \gamma_{5} \frac{T_{\alpha}}{2} S^{i} g_{\mathrm{P} q q} \gamma_{5} T_{\beta}^{\dagger} S^{j}\right]
\end{aligned}
$$

where the meson-quark-quark effective coupling $g_{\mathrm{P} q q}$ is defined by

$$
\left(g_{\mathrm{P} q q}\right)^{-2}=M_{0}^{4-D} \frac{\partial \Pi_{\mathrm{P}}\left(k^{2}\right)}{\partial k^{2}} .
$$

For notational simplicity, when it is obvious, we will omit the subscript "rs" indicating that the quantity is rescaled with the help of a power of $M_{0}$. In the leading order of $1 / N_{c}$ expansion, the decay constants are calculated as [16],

$$
\begin{aligned}
f_{\pi}^{2}= & m_{u}^{* 2} M_{0}^{4-D} I_{u u}(0), \\
f_{\mathrm{K}}^{2}= & \frac{M_{0}^{4-D}}{J_{u s}(0)}\left[m_{u}^{*} I_{u s}(0)+\left(m_{s}^{*}-m_{u}^{*}\right)\right. \\
& \left.\times \operatorname{tr} \int_{0}^{1} d x \int \frac{d^{D} p}{i(2 \pi)^{D}} \frac{x}{\left\{p^{2}-L_{u s}(0)+i \varepsilon\right\}^{2}}\right]^{2},
\end{aligned}
$$

where $J_{u s}$ is defined by

$$
\begin{aligned}
& J_{u s}\left(k^{2}\right)=I_{i j}\left(k^{2}\right)+2\left\{\left(m_{s}^{*}-m_{u}^{*}\right)^{2}-k^{2}\right\} \\
& \quad \times \operatorname{tr} \int_{0}^{1} d x \int \frac{d^{D} p}{i(2 \pi)^{D}} \frac{x(1-x)}{\left\{p^{2}-L_{u s}\left(k^{2}\right)+i \varepsilon\right\}^{3}},
\end{aligned}
$$

with

$$
L_{i j}\left(k^{2}\right)=m_{i}^{* 2}-\left(m_{i}^{* 2}-m_{j}^{* 2}\right) x-k^{2} x(1-x) .
$$

Equation (25) is used to fix the mass scale parameter $M_{0}$.

\section{Topological susceptibility}

The topological susceptibility $\chi$ is defined by the correlation function between the topological charge densities, $Q(x)$, at different points [4],

$$
\chi=\int d^{D} x\langle 0|T Q(x) Q(0)| 0\rangle_{\text {connected }}
$$

where

$$
Q(x) \equiv \frac{g^{2}}{32 \pi^{2}} F_{\mu \nu}^{a} \tilde{F}^{a \mu \nu}=2 K \operatorname{Im}\left[\operatorname{det} \bar{q}\left(1-\gamma_{5}\right) q\right]
$$

$g$ is the strong coupling constant of QCD and $F_{\mu \nu}^{a}$ is the field strength for gluons. Equation (28) should be 
multiplied by $M_{0}^{4-D}$ to adjust the mass dimensions. In the leading order of $1 / N_{c}$ expansion $\chi$ is given by [26]

$$
\begin{aligned}
\chi= & \frac{4 K^{2}}{M_{0}^{D-4}}\left(i \operatorname{tr} S^{u}\right)^{2}\left[\left(i \operatorname{tr} S^{u}\right)\left(i \operatorname{tr} S^{s}\right)\left(\frac{2 i \operatorname{tr} S^{s}}{m_{u}^{*}}+\frac{i \operatorname{tr} S^{u}}{m_{s}^{*}}\right)\right. \\
& +\left\{\frac{1}{\sqrt{6}}\left(2 i \operatorname{tr} S^{s}+i \operatorname{tr} S^{u}\right)\left(\Pi_{00}(0), \Pi_{08}(0)\right)\right. \\
& \left.+\frac{1}{\sqrt{3}}\left(i \operatorname{tr} S^{s}-i \operatorname{tr} S^{u}\right)\left(\Pi_{08}(0), \Pi_{88}(0)\right)\right\} \boldsymbol{\Delta}^{+}(0) \\
& \times\left\{\frac{1}{\sqrt{6}}\left(2 i \operatorname{tr} S^{s}+i \operatorname{tr} S^{u}\right)\left(\begin{array}{c}
\Pi_{00}(0) \\
\Pi_{08}(0)
\end{array}\right)\right. \\
& \left.\left.+\frac{1}{\sqrt{3}}\left(i \operatorname{tr} S^{s}-i \operatorname{tr} S^{u}\right)\left(\begin{array}{c}
\Pi_{08}(0) \\
\Pi_{88}(0)
\end{array}\right)\right\}\right]
\end{aligned}
$$

Thus $\chi$ is evaluated by the quantities already obtained above.

\section{STRATEGY}

As mentioned in the introduction, our goal is to test the regularization parameter dependence (or independence) in the NJL model.

We use the abstract denotations: $\mathrm{m}(\in \mathcal{M})$ are the model predictions, $\mathrm{p}(\in \mathcal{P})$ are the model parameters, and $\mathrm{i}(\in \mathcal{I})$ are the input physical quantities. The capitals $\mathcal{M}, \mathcal{P}$ and $\mathcal{I}$ represent the sets of these quantities. The model predictions can be performed if all the parameters are known, so the model determines some function $\mathcal{F}_{\text {mp }}$,

$$
\mathrm{m}^{\mathrm{R}}(\mathcal{P})=\mathcal{F}_{\mathrm{mp}}^{\mathrm{R}}(\mathcal{P})
$$

where the superscript $\mathrm{R}$ indicates a regularization procedure. In the similar manner one can obtain the functions $\mathcal{F}_{\text {pi }}^{\mathrm{R}}$ which connect $\mathcal{P}$ and $\mathcal{I}$ through the parameter fitting. Thus, the model relates $\mathcal{I}$ to $\mathcal{M}$, as

$$
\mathrm{m}^{\mathrm{R}}(\mathcal{I})=\mathcal{F}_{\mathrm{mi}}^{\mathrm{R}}(\mathcal{I})
$$

The resulting values of $\mathrm{m}$ should not depend on regularization methods, if they correctly capture the physics in question. In the next section, we obtain these functions $\mathcal{F}_{\text {mi }}^{\mathrm{R}}, \mathcal{F}_{\mathrm{mp}}^{\mathrm{R}}$ and $\mathcal{F}_{\mathrm{pi}}^{\mathrm{R}}$.

Our model with the DR has six free parameters $\mathcal{P}_{6}=\left\{m_{u}, m_{s}, G, K, D, M_{0}\right\}$. Four of them, $\mathcal{P}_{4}=$ $\left\{m_{s}, G, K, M_{0}\right\}$, may be fitted to the input meson properties, $\mathcal{I}_{4}=\left\{m_{\pi}, f_{\pi}, m_{\mathrm{K}}, m_{\eta^{\prime}}\right\}$. After this partial parameter setting, predicted meson properties, $\mathcal{M}=$ $\left\{f_{\mathrm{K}}, m_{\eta}, \chi, \ldots\right\}$ are written as the functions of the remaining parameters $m_{u}$ and $D$,

$$
\mathrm{m}^{\mathrm{DR}}\left(m_{u}, D\right)=\left.\mathcal{F}_{\mathrm{mp}}^{\mathrm{DR}}\left(m_{u}, D\right)\right|_{\mathcal{P}_{4} \leftarrow \mathcal{I}_{4}} .
$$

We cannot obtain finite values for all predicted quantities in the $4 \mathrm{D}$ limit, all of $\mathrm{m}$ are finite at $D<4$. However, it is possible to obtain finite values for some of $m$ at the four dimensional limit. Furthermore, some of physical quantities may be independent of the parameter $D$ [16]. We are interested in this aspect of the NJL model which does not depend on the regularization parameter. Namely, it is interesting that in the model discussed here there are theoretical relations between some observables which are finite (although the model is not renormalizable) and, moreover, they do not depend on the regularization parameter. We mean the relations between input and predicted observables derived with the help of the fitting parameters procedure.

Once we put the actual numbers into $\mathcal{I}_{4}$ and fix $D$, $\mathrm{m}$ become the functions of $m_{u}$. In particular, we shall evaluate them in the $D \rightarrow 4$ limit, then compare between each other results obtained in the three regularization ways: (1) DR with $D \rightarrow 4$, (2) DR with fixed $D$ by $\chi$, and (3) three-momentum (3M) cutoff method. These regularization schemes can symbolically be written as

$$
\begin{aligned}
& (1) \mathrm{m}^{4 \mathrm{D}}\left(m_{u}\right)=\left.\mathcal{F}_{\mathrm{mp}}^{\mathrm{DR}}\left(m_{u}\right)\right|_{\mathcal{P}_{4} \leftarrow \mathcal{I}_{4}, D \rightarrow 4}, \\
& (2) \mathrm{m}^{\mathrm{DR}}\left(m_{u}\right)=\left.\mathcal{F}_{\mathrm{mp}}^{\mathrm{DR}}\left(m_{u}\right)\right|_{\left\{\mathcal{P}_{4}, D\right\} \leftarrow\left\{\mathcal{I}_{4}, \chi\right\}}, \\
& (3) \mathrm{m}^{3 \mathrm{M}}\left(m_{u}\right)=\left.\mathcal{F}_{\mathrm{mp}}^{3 \mathrm{M}}\left(m_{u}\right)\right|_{\mathcal{P}_{4}^{3 \mathrm{M}} \leftarrow \mathcal{I}_{4}},
\end{aligned}
$$

where $\mathcal{P}_{4}^{3 \mathrm{M}}=\left\{m_{s}, G, K, \Lambda\right\}$, with the momentum cutoff $\Lambda$. Note that the total number of the parameters in the cutoff method is five [16], since this method does not need the mass parameter.

\section{FOUR DIMENSIONAL LIMIT}

We demonstrate the calculation in the four dimensional (4D) limit and express the predicted physical quantities in terms of the input meson properties. By virtue of taking the $4 \mathrm{D}$ limit, the relations among the physical quantities can be simplified through the leading order of $\epsilon$ expansion. It is even possible to obtain analytic expressions for some quantities, which will be discussed below.

\section{A. The summary of the Sec. III}

To make the model prediction we need to know the following six quantities:

$$
m_{u}^{*}, m_{s}^{*}, G, K, m_{s}, M_{0}
$$

These are evaluated by the gap equations

$$
\begin{aligned}
& m_{u}^{*}=m_{u}+4 G\left(i \operatorname{tr} S^{u}\right)+2 K\left(i \operatorname{tr} S^{u}\right)\left(i \operatorname{tr} S^{s}\right), \\
& m_{s}^{*}=m_{s}+4 G\left(i \operatorname{tr} S^{s}\right)+2 K\left(i \operatorname{tr} S^{u}\right)^{2},
\end{aligned}
$$

and the conditions for meson properties

$$
\begin{aligned}
& 1-2 K_{3} \Pi_{\pi}\left(m_{\pi}^{2}\right)=0, \\
& 1-2 K_{6} \Pi_{\mathrm{K}}\left(m_{\mathrm{K}}^{2}\right)=0, \\
& \operatorname{det}\left[1-2 \boldsymbol{K}^{+} \boldsymbol{\Pi}\left(m_{\eta^{\prime}}^{2}\right)\right]=0, \\
& f_{\pi}^{2}=m_{u}^{* 2} M_{0}^{4-D} I_{u u}(0) .
\end{aligned}
$$

In the following, we will calculate the quantities (34) by solving these six equations in the $4 \mathrm{D}$ limit. 


\section{B. Constituent quark masses $m_{u}^{*}, m_{s}^{*}$}

The constituent quark mass $m_{u}^{*}$ is obtained from Eqs. (35) and (37). The gap equation (35) can be rewritten as

$$
m_{u}^{*}=m_{u}+4 K_{3}\left(i \operatorname{tr} S^{u}\right)
$$

which in combination with Eq. (37) enables one to get rid of $K_{3}$ and thereby to calculate $m_{u}^{*}$ without knowing the values of $G$ and $K$.

In the $4 \mathrm{D}(\epsilon \rightarrow 0)$ limit, the chiral condensate $i \operatorname{tr} S^{i}$ and the self-energy loop integral $\Pi_{5}^{i j}\left(k^{2}\right)$ can be expanded in powers of $\epsilon$ as

$$
\begin{aligned}
& i \operatorname{tr} S^{i}=-\frac{N_{c}}{4 \pi^{2} \epsilon} m_{i}^{* 3}, \\
& \Pi_{5}^{i j}\left(k^{2}\right)=\frac{N_{c}}{8 \pi^{2} \epsilon}\left[k^{2}-2\left(m_{i}^{* 2}+m_{j}^{* 2}-m_{i}^{*} m_{j}^{*}\right)\right] .
\end{aligned}
$$

After some algebra we arrive at

$$
m_{u}^{*}=-\frac{m_{\pi}^{2}}{4 m_{u}}\left\{1+\sqrt{1+\frac{8 m_{u}^{2}}{m_{\pi}^{2}}}\right\} .
$$

Here we adopted the negative solution of $m_{u}^{*}$, since the positive solution is unstable.

We thus find the analytic expression for $m_{u}^{*}$ as a function of $m_{\pi}$. On the other hand we apply numerical analysis to obtain $m_{s}^{*}$ by simultaneously solving Eqs. (37), (38) and (39).

\section{Couplings $G, K$}

The couplings $G$ and $K$ can be written as the functions of $m_{u}^{*}, m_{s}^{*}, m_{\pi}$ and $m_{\mathrm{K}}$.

From Eqs. (37) and (38), one derives the following relations

$$
\begin{aligned}
G & =\frac{-i \operatorname{tr} S^{u} \Pi_{\mathrm{K}}+i \operatorname{tr} S^{s} \Pi_{\pi}}{2\left(i \operatorname{tr} S^{s}-i \operatorname{tr} S^{u}\right) \Pi_{\pi} \Pi_{\mathrm{K}}}, \\
K & =\frac{\Pi_{\mathrm{K}}-\Pi_{\pi}}{\left(i \operatorname{tr} S^{s}-i \operatorname{tr} S^{u}\right) \Pi_{\pi} \Pi_{\mathrm{K}}} .
\end{aligned}
$$

where we use the abbreviated notations of $\Pi_{\pi}=\Pi_{\pi}\left(m_{\pi}^{2}\right)$ and $\Pi_{\mathrm{K}}=\Pi_{\mathrm{K}}\left(m_{\mathrm{K}}^{2}\right)$. By using Eqs. (13), (14) and (43) we arrive at the following expressions for the couplings

$$
\begin{aligned}
G= & \frac{2 \pi^{2} \epsilon}{N_{c}} \frac{1}{m_{s}^{* 3}-m_{u}^{* 3}}\left[\frac{m_{s}^{* 3}}{m_{\mathrm{K}}^{2}-2\left(m_{u}^{* 2}+m_{s}^{* 2}-m_{u}^{*} m_{s}^{*}\right)}\right. \\
& \left.-\frac{m_{u}^{* 3}}{m_{\pi}^{2}-2 m_{u}^{* 2}}\right]+\mathcal{O}\left(\epsilon^{2}\right), \\
K= & \frac{16 \pi^{4} \epsilon^{2}}{N_{c}^{2}} \frac{1}{m_{s}^{* 3}-m_{u}^{* 3}}\left[\frac{1}{m_{\mathrm{K}}^{2}-2\left(m_{u}^{* 2}+m_{s}^{* 2}-m_{u}^{*} m_{s}^{*}\right)}\right. \\
& \left.-\frac{1}{m_{\pi}^{2}-2 m_{u}^{* 2}}\right]+\mathcal{O}\left(\epsilon^{3}\right) .
\end{aligned}
$$

Thus the couplings are the functions of $m_{s}^{*}, G\left(m_{s}^{*}\right)$ and $K\left(m_{s}^{*}\right)$. With the help of Eq. (39), we can determine $m_{s}^{*}$ and substitute it in $G\left(m_{s}^{*}\right)$ and $K\left(m_{s}^{*}\right)$ to get $G$ and $K$.

Note that $G$ and $K$ are of the order of $\epsilon$ and $\epsilon^{2}$, respectively. In the naive $\epsilon \rightarrow 0$ limit $G$ and $K$ tend to 0 [24]. However, the combinations $G \operatorname{tr} S$ and $K(\operatorname{tr} S)^{2}$ appears to be finite because the chiral condensate, Eq. (42), is of the order of $\epsilon^{-1}$. Then, the dynamical masses, Eq. (4), can have larger values than $m_{i}$ even in the limit of $\epsilon \rightarrow 0$.

From the order counting of $1 / \epsilon$ for Eqs. (35), (36) and (42), we find the following relation,

$$
\left(\frac{1}{\epsilon}\right)^{L-N_{G}-2 N_{K}}=\left(\frac{1}{\epsilon}\right)^{1-n / 2},
$$

where $L, N_{G}$ and $N_{K}$ are the number of quark loops (chiral condensates), $G$ and $K$, respectively. $n$ is the number of the external quark lines $(n=2 l, l=0,1,2, \cdots, l$ are the number of external meson lines). Then $n$ point functions become finite except the bubble diagrams.

For instance, the above discussion is manifested in the expressions for the pion and kaon propagators in the limit $D \rightarrow 4$

$$
\Delta_{\mathrm{P}}\left(k^{2}\right)=-\frac{4 \pi^{2} \epsilon}{N_{c}} \frac{1}{k^{2}-m_{\mathrm{P}}^{2}} .
$$

These are derived through the substitution of the obtained $G, K$ and $\Pi_{\mathrm{P}}$ into Eq. (10).

\section{Current strange quark mass $m_{s}$}

We have seen that $m_{u}^{*}, m_{s}^{*}, G$ and $K$ are determined from Eqs. (35), (37), (38) and (39). Substituting these into Eq. (36), it is easy to evaluate $m_{s}$. The numerical result will be shown in Sec. VIB.

\section{E. Mass scale parameter $M_{0}$}

In this subsection, we shall set the remaining parameter $M_{0}$, the mass scale, by using the pion decay constant $f_{\pi}$ in Eq. (40).

In the $4 \mathrm{D}$ limit, the integral $I_{u u}$ (Eq. (16) ) becomes

$$
\begin{aligned}
\lim _{\epsilon \rightarrow 0} I_{u u} & =\lim _{\epsilon \rightarrow 0} \frac{N_{c}}{(2 \pi)^{2-\epsilon}} \Gamma(\epsilon) \int_{0}^{1} d x L_{i j}^{-\epsilon} \\
& \simeq-\lim _{\epsilon \rightarrow 0} \frac{N_{c}}{4 \pi^{2} \epsilon} .
\end{aligned}
$$

Hence we obtain the mass scale from Eq. (25):

$$
\lim _{\epsilon \rightarrow 0} M_{0}^{2 \epsilon}=\lim _{\epsilon \rightarrow 0} \frac{4 \pi^{2} \epsilon}{N_{c}} \frac{f_{\pi}^{2}}{m_{u}^{* 2}} .
$$

Since $m_{u}^{*}$ is the function of $m_{\pi}$, (44), the value of $M_{0}$ is determined by $m_{\pi}$ and $f_{\pi} . M_{0}$ goes to 0 in the limit of $\epsilon \rightarrow 0$. 
It is worth mentioning that by using Eqs. (52), (26) and (27), $f_{\mathrm{K}}$ can be written as

$$
f_{\mathrm{K}}^{2}=f_{\pi}^{2} \frac{\left(m_{u}^{*}+m_{s}^{*}\right)^{2}}{4 m_{u}^{* 2}}
$$

since $J_{i j}$ coincides with $I_{i j}$ in the leading order of the $\epsilon$ expansion.

\section{F. Chiral condensates $\langle\bar{u} u\rangle,\langle\bar{s} s\rangle$}

From the Eq. (42) the chiral condensates $\langle\bar{i} i\rangle$ are of the order of $\epsilon^{-1}$, so they diverge in the $\epsilon \rightarrow 0$ limit. However, the rescaled chiral condensates $\langle\bar{i} i\rangle_{\mathrm{rs}}\left(\equiv M_{0}^{4-D}\langle\bar{i} i\rangle\right)$ are finite, since the order of $M_{0}^{4-D}$ is $\epsilon$ as seen in Eq. (52).

The form of the rescaled chiral condensate $\langle\bar{u} u\rangle_{\mathrm{rs}}$ is obtained from Eqs. (42), (44) and (52) as

$$
\langle\bar{u} u\rangle_{\mathrm{rs}}=-\frac{m_{\pi}^{2} f_{\pi}^{2}}{4 m_{u}}\left\{1+\sqrt{1+\frac{8 m_{u}^{2}}{m_{\pi}^{2}}}\right\} .
$$

If we ignore the last term of Eq. (54), the above equation coincides with the Gell-Mann-Oakes-Renner relation [28, 29]. With the help of Eq. (53), one has the analytic expression for $\langle\bar{s} s\rangle_{\mathrm{rs}}$ :

$$
\langle\bar{s} s\rangle_{\mathrm{rs}}=-\frac{m_{\pi}^{2} f_{\pi}^{2}}{4 m_{u}}\left(2 \frac{f_{\mathrm{K}}}{f_{\pi}}-1\right)^{3}\left\{1+\sqrt{1+\frac{8 m_{u}^{2}}{m_{\pi}^{2}}}\right\} .
$$

In the limit $f_{\mathrm{K}} \rightarrow f_{\pi},\langle\bar{s} s\rangle_{\mathrm{rs}}$ coincides with $\langle\bar{u} u\rangle_{\mathrm{rs}}$.

\section{NUMERICAL RESULTS}

To evaluate the physical quantities discussed in Sec.III we employ the following input meson properties:

$$
\begin{aligned}
& m_{\pi}=138 \mathrm{MeV}, f_{\pi}=92 \mathrm{MeV}, \\
& m_{\mathrm{K}}=495 \mathrm{MeV}, m_{\eta^{\prime}}=958 \mathrm{MeV} .
\end{aligned}
$$

We first evaluate the constituent quark masses and the strange current quark mass, then calculate the meson properties $f_{\mathrm{K}}, m_{\eta}$, and the topological susceptibility $\chi$.

As mentioned in Sec. IV we compare the results obtained in the the three cases: (1) DR with 4D, (2) DR with $D(\chi)$, and (3) $3 \mathrm{M}$ cutoff method. In the second case, we select the value $\chi^{1 / 4}=170 \mathrm{MeV}$ [16] and call it simply the DR results.

\section{A. Constituent quark masses $m_{u}^{*}, m_{s}^{*}$}

Before evaluating the observed quantities, we consider an unobserved quantity, the constituent quark mass. In Fig. 1) $m_{u}^{*}$ and $m_{s}^{*}$ are shown as the functions of $m_{u}$ in the range $3 \mathrm{MeV} \leq m_{u} \leq 6 \mathrm{MeV}$. Since $m_{u}$ is contained in the denominator of Eq. (44), $m_{u}^{*}$ depends strongly on

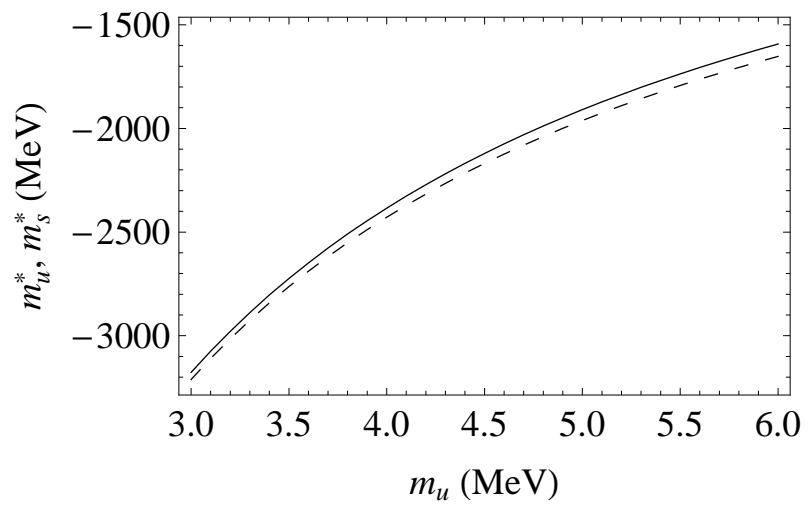

FIG. 1. $m_{u}^{*}$ and $m_{s}^{*}$ as the functions of $m_{u}$. The solid and dashed lines are the results of $m_{u}^{*}$ and $m_{s}^{*}$, respectively.

$m_{u}$. Note that $m_{u}^{*}$ and $m_{s}^{*}$ can be positive according the discussion in Ref. [18]. The values of $\left|m_{u}^{*}\right|$ and $\left|m_{s}^{*}\right|$ are considerably larger than in the frequently used $3 \mathrm{M}$ cutoff case, $m_{u}^{*} \sim 300 \mathrm{MeV}$ and $m_{s}^{*} \sim 500 \mathrm{MeV}$. Therefore a large regularization dependence is found for an unobserved quantity. Note that $m_{s}^{*}$ can be obtained by solving Eq. (53) analytically. However, this solution does not lead a realistic value of $m_{\eta^{\prime}}$. In other words the observed value of the kaon decay constant, $f_{\mathrm{K}}$, can not be consistent with the realistic value for $m_{\eta^{\prime}}$.

\section{B. Current quark mass $m_{s}$}

The plots of $m_{s}$ obtained in the $4 \mathrm{D}, \mathrm{DR}$ and cutoff cases are shown in Fig. 2. We see that $m_{s}$ increases

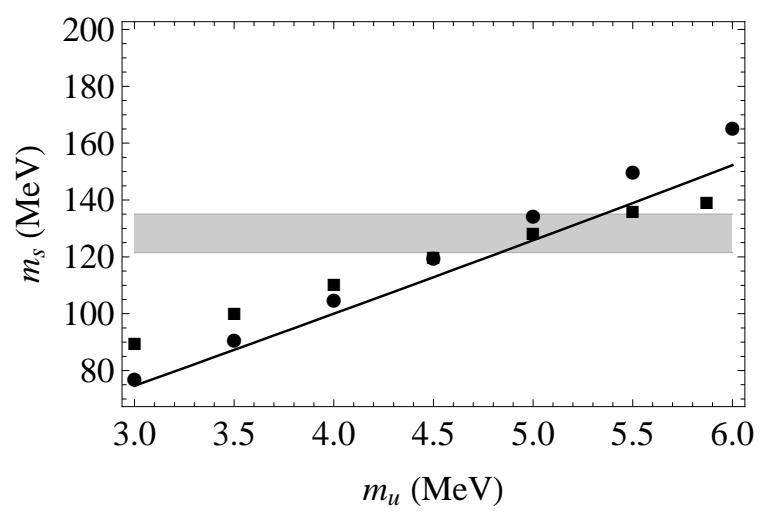

FIG. 2. The strange quark mass $m_{s}$ as the function of $m_{u}$. The black line shows $m_{s}$ in the $4 \mathrm{D}$ case. The circles and squares are DR and cutoff regularization results, respectively. The gray bound is the experimental region.

linearly with respect to $m_{u}$. It is interesting to note that the DR and cutoff results show behavior similar to the $4 \mathrm{D}$ result. The results around $m_{u} \simeq 5 \mathrm{MeV}$ cross the experimental region which is evaluated at $1 \mathrm{GeV}[27]$. 


\section{Kaon decay constant $f_{\mathrm{K}}$}

Figure 3 displays the results of $f_{\mathrm{K}}$ with the experimental value $f_{\mathrm{K}}^{\mathrm{ex}}=110 \mathrm{MeV}$. The resulting $f_{\mathrm{K}}$ in the $4 \mathrm{D}$ case

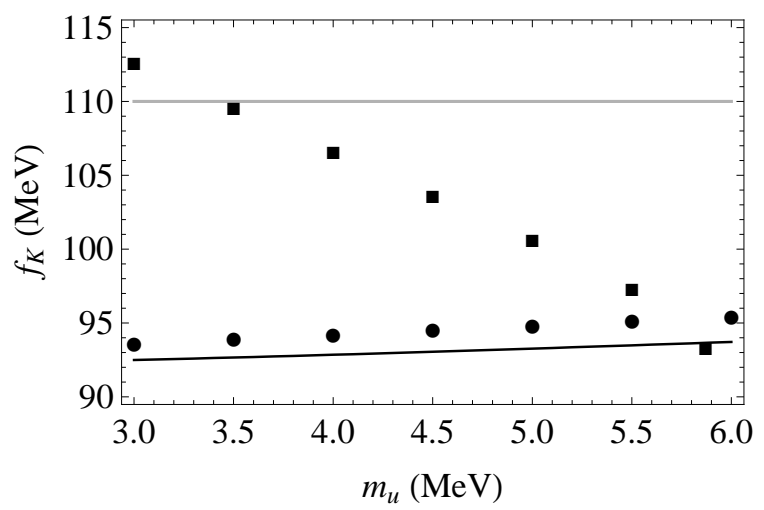

FIG. 3. The kaon decay constant $f_{\mathrm{K}}$ as the function of $m_{u}$. Black and gray lines are the obtained $f_{\mathrm{K}}$ and the experimental value. The circles and squares are DR and cutoff regularization results, respectively.

is smaller than its experimental value. The DR plots are similar to the $4 \mathrm{D}$ case, and they are a few $\mathrm{MeV}$ closer to the experimental line. On the other hand, $f_{\mathrm{K}}$ decreases with increasing $m_{u}$ in the cutoff regularization, which is the opposite to the 4D and DR cases tendency. The cutoff results for small $m_{u}$ region receive the large effect of the parameter dependence.

\section{Eta meson mass $m_{\eta}$}

We put the result of $m_{\eta}$ in Fig. 4. In the $4 \mathrm{D}$ case, $m_{\eta}$

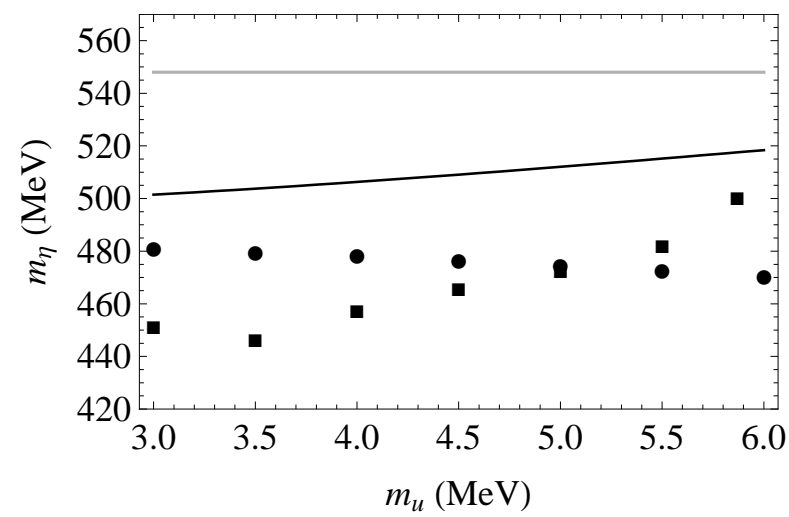

FIG. 4. The eta meson mass $m_{\eta}$ as the function of $m_{u}$. The black and gray lines are the obtained $m_{\eta}$ and the experimental value, $m_{\eta}^{\mathrm{ex}}=548 \mathrm{MeV}$. The circles and squares are the DR and the cutoff results.

is around $500 \mathrm{MeV}$ at $m_{u}=3.0 \mathrm{MeV}$, and it slightly increases with respect to $m_{u}$. For all the region, the values are smaller than in the experimental data. Contrary to the results seen in $f_{\mathrm{K}}$, both the DR and cutoff cases are worse than the $4 \mathrm{D}$ case in terms of comparison with the experimental data.

\section{E. Topological susceptibility $\chi$}

In Fig. 5. $\chi$ is calculated as a function of $m_{u}$. One sees

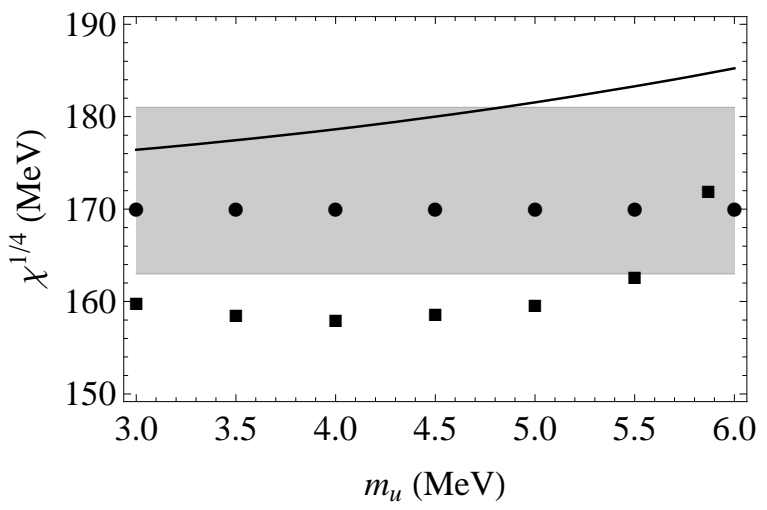

FIG. 5. The topological susceptibility $\chi$ as the function of $m_{u}$. The black line is the obtained $\chi$ in the $4 \mathrm{D}$. The circles and squares are the DR and cutoff results, respectively. The gray bound is the result of lattice simulation [30].

that, in the 4D case, $\chi$ becomes larger when $m_{u}$ increases. The DR result is trivially fixed at $\chi^{1 / 4}=170 \mathrm{MeV}$, because $\chi$ is the fifth input quantity in this case. The gray bound shows the results of lattice simulation [30], $\chi^{1 / 4}=170 \pm 7,174 \pm 7 \mathrm{MeV}$, and Witten-Veneziano mass formula [31, 32], $\chi^{1 / 4}=179 \mathrm{MeV}$. The $4 \mathrm{D}$ result is close to the values of lattice simulation. The $4 \mathrm{D}$ and $\mathrm{DR}$ results are plotted inside the lattice region. However, almost all squares are located outside of the lattice region, so the cutoff case is worse than the $4 \mathrm{D}$ and $\mathrm{DR}$ in this context.

\section{F. Chiral condensates $\langle\bar{u} u\rangle,\langle\bar{s} s\rangle$}

The chiral condensates are shown as functions of $m_{u}$ in Fig. 6] where we use the obtained $f_{\mathrm{K}}$ to evaluate Eq. (55). The $m_{u}$ dependence can easily be read off the explicit forms in Eqs. (54) and (55). It is interesting to note that, although the values of $\left|m_{i}^{*}\right|$ in the $4 \mathrm{D}$ and DR methods are much larger than in the cutoff case, $m_{i}^{*} \sim \mathcal{O}(100 \mathrm{MeV})$, the chiral condensates almost coincide in the $4 \mathrm{D}$ and $\mathrm{DR}$ regularizations.

\section{CONCLUSION}

We have constructed a NJL model with DR which is convergent in the 4D limit, and found that it is possible to control the ultraviolet divergences within the framework mentioned in the section $\mathrm{V}$. The obtained results describe meson properties pretty well. We believe that 


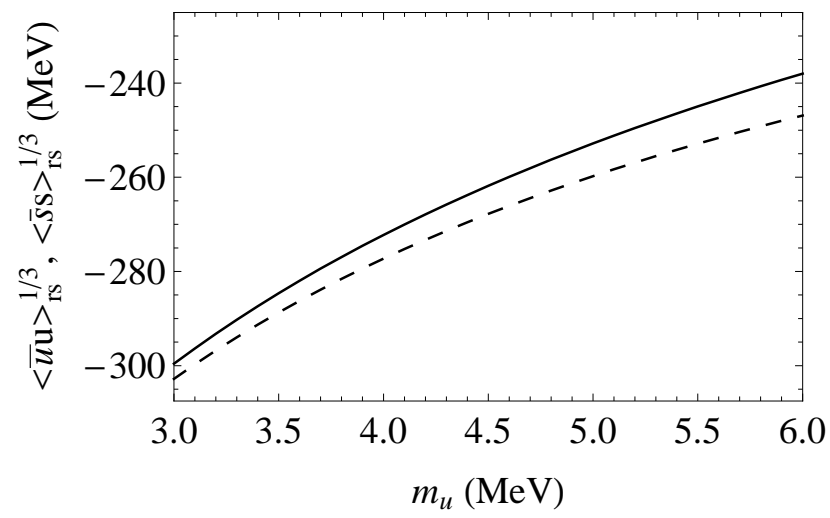

FIG. 6. The chiral condensates $\langle\bar{u} u\rangle_{\mathrm{rs}}$ and $\langle\bar{s} s\rangle_{\mathrm{rs}}$ as the function of $m_{u}$. The solid and dashed lines indicate $\langle\bar{u} u\rangle_{\mathrm{rs}}$ and $\langle\bar{s} s\rangle_{\mathrm{rs}}$, respectively.

the treatment prescribed here has possibilities for future work.

(I) We have shown that the model is free of divergences by virtue of introducing the mass scale parameter. The mass scale runs according the dimension in the dimensional regularization scheme where the ultraviolet limit corresponds to $4 \mathrm{D}$ limit. We find that it is possible to remove all the divergence within the model frame work. Here the mass-scaling determines the scale of the model, so this is reminiscent of the renormalization flow. We have explicitly shown the ultraviolet behavior via numerical calculations.

(II) It is a non-trivial question whether so constructed model can produce reliable results, because important contributions may be dropped due to taking the $4 \mathrm{D}$ limit. However the obtained results indicate that the model behaves well even in this limit.

(III) The meson properties are described without the regularization parameter. The model predictions do not depend on $D$ due to the effect of the mass rescaling. This indicates that we can consider the ultraviolet limit even in the nonrenormalizable model.

(IV) We found that, in this model framework, it is possible to obtain analytical relations between meson properties and the chiral condensates. This point has practical importance as the model calculations are significantly simplified. In particular, Eq. (50) has quite simple form for the meson propagators. This form implies that we can introduce meson propagators in the same form as it appears in the usual perturbative expansion techniques. Therefore the method is expected to be useful in more complicated problems such as for example in the threebody formalism.

We have also clarified the following less important points:

(V) By applying the effects of the mass scaling, we have clarified the role of the mass dimension. It is known that the couplings should become zero in the ultraviolet limit so that the theory well behaves in the ultraviolet limit [6]. We have effectively incorporated the "running" couplings by using the mass rescaling to control the divergences with keeping the finite values of the rescaled couplings.

(VI) Related to the point (I) we have introduced the effective coupling scale in the couplings $G$ and $K$, and this works successfully. This is trivial if one deals with the renormalized theory where the ultraviolet behavior is well known through the renormalization group flow. However, in the NJL model, the background gluon contributions are implicitly expressed in terms of the effective coupling strength. Therefore the couplings regularized in specific ways contain important dynamical information of the model. Our results indicate that the model with the DR does not miss dominant physical contributions when we take the high-energy limit.

The model predictions are intimately related to the employed regularization prescriptions, because they include the background dynamical information as was explained in the point (VI). Then, it may be interesting to investigate whether the ultraviolet behavior of the model different regularization schemes leads to the similar consequences. We believe the DR method is especially good in this context, because it is expected to preserve the required symmetry of the model just as it does quite successfully in the formal perturbative quantum field theories. This statement is confirmed by phenomenological results of the paper.

Concerning the point (II) we also found that the physical quantities calculated in the DR with $D<4$ and in the cutoff regularization have values similar to those obtained in the DR at the $4 \mathrm{D}$ limit.

However, there is a discrepancy between the model results and the experimentally observed values. We believe that this discrepancy should be explained by introducing higher dimensional operators.

Calculations are drastically simplified if one uses our method thanks to taking the $4 \mathrm{D}$ limit. Thus we hope that the regularization parameter independent approach discussed in this paper can be useful to systematically introduce higher dimensional operators and to calculate higher order corrections.

\section{ACKNOWLEDGMENTS}

The authors would like to thank Y. Hoshino, T. Morozumi and K. Ishikawa for fruitful discussions. HK is supported by the National Research Foundation of Korea funded by the Korean Government (Grant No. NRF-2011-220-C00011). AK is supported by the Georgian Shota Rustaveli National Science Foundation (grant 11/31). 
[1] Y. Nambu and G. Jona-Lasinio, Phys. Rev. 122, 345 (1961); 124, 246 (1961).

[2] U. Vogl and W. Weise, Prog. Part. Nucl. Phys. 27, 195 (1991)

[3] S. P. Klevansky, Rev. Mod. Phys. 64, 649 (1992).

[4] T. Hatsuda and T. Kunihiro, Phys. Rept. 247, 221 (1994).

[5] D. J. Gross and A. Neveu, Phys. Rev. D 10, 3235 (1974).

[6] T. Eguchi, Phys. Rev. D 17, 611 (1978).

[7] K. -i. Shizuya, Phys. Rev. D 21, 2327 (1980).

[8] B. Rosenstein, B. J. Warr and S. H. Park, Phys. Rev. Lett. 62, 1433 (1989).

[9] R.S. Plant and M.C. Birse, Nucl. Phys. A 628, 607 (1998); A 703, 717 (2002).

[10] T. Hell, S. Roessner, M. Cristoforetti and W. Weise, Phys. Rev. D 79, 014022 (2009); D 81, 074034 (2010).

[11] S. Krewald and K. Nakayama, Ann. Phys. 216, 201 (1992).

[12] T. Inagaki, T. Kouno and T. Muta, Int. J. Mod. Phys. A 10, 2241 (1995).

[13] R.G. Jafarov, and V.E. Rochev Russ. Phys. J. 49, 712 (2006).

[14] T. Inagaki and D. Kimura and A. Kvinikhidze, Phys. Rev. D 77, 116004 (2008).

[15] T. Fujihara, D. Kimura, T. Inagaki and A. Kvinikhidze Phys. Rev. D 79, 096008 (2009).

[16] T. Inagaki, D. Kimura, H. Kohyama and A. Kvinikhidze, Phys. Rev. D 83, 034005 (2011).

[17] T. Inagaki, D. Kimura, H. Kohyama and A. Kvinikhidze, Phys. Rev. D 85, 076002 (2012).
[18] T. Inagaki, D. Kimura, H. Kohyama and A. Kvinikhidze, Phys. Rev. D 86, 116013 (2012).

[19] A.A. Osipov, A.H. Blin and B. Hiller, arXiv:hep-ph/0410148 A.A. Osipov, H. Hansen and B. Hiller, Nucl. Phys. A 745, 81 (2004).

[20] T. Inagaki, S.D. Odintsov and Yu.I Shil'nov, Int. J. Mod. Phys. A 14, 481 (1999).

[21] T. Inagaki, D. Kimura and T. Murata, Prog. Theor. Phys. 111, 371 (2004).

[22] M. Kobayashi and T. Maskawa, Prog. Theor. Phys. 44, 1422 (1970); M. Kobayashi, H. Kondo and T. Maskawa, Prog. Theor. Phys. 45, 1955 (1971).

[23] G. 't Hooft, Phys. Rev. D 14, 3432 (1976); 18, 2199(E) (1978); Phys. Rept. 142, 357 (1986).

[24] K. G. Wilson, Phys. Rev. D 7, 2911 (1973).

[25] P. Rehberg, S. P. Klevansky and J. Hufner, Phys. Rev. C 53, 410 (1996).

[26] K. Fukushima, K. Ohnishi and K. Ohta, Phys. Rev. C 63, 045203 (2001).

[27] J. Beringer et al. [Particle Data Group Collaboration], Phys. Rev. D 86, 010001 (2012).

[28] M. Gell-Mann, R. J. Oakes and B. Renner, Phys. Rev. 175, 2195 (1968).

[29] S. L. Glashow and S. Weinberg, Phys. Rev. Lett. 20, 224 (1968).

[30] B. Alles, M. D'Elia and A. Di Giacomo, Nucl. Phys. B 494, 281 (1997); B 679, 397(E) (2004).

[31] E. Witten, Nucl. Phys. B 156, 269 (1979).

[32] G. Veneziano, Nucl. Phys. B 159, 213 (1979). 